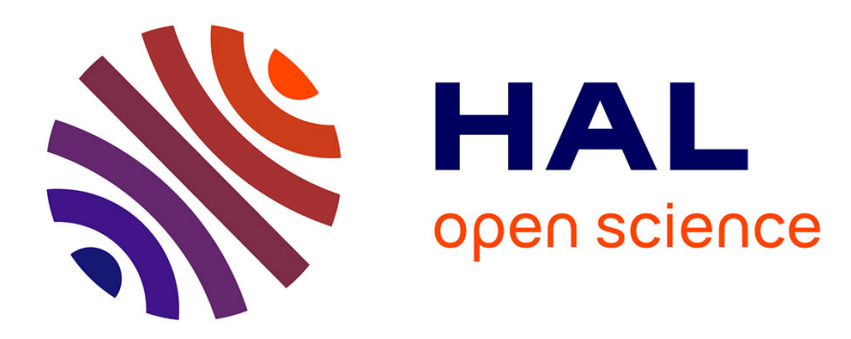

\title{
Les étudiants et le Contrat première embauche
}

Sébastien Michon

\section{- To cite this version:}

Sébastien Michon. Les étudiants et le Contrat première embauche. Agora débats/jeunesses, 2008, 49, p. 64-76. halshs-00368292

\section{HAL Id: halshs-00368292 \\ https://shs.hal.science/halshs-00368292}

Submitted on 16 Mar 2009

HAL is a multi-disciplinary open access archive for the deposit and dissemination of scientific research documents, whether they are published or not. The documents may come from teaching and research institutions in France or abroad, or from public or private research centers.
L'archive ouverte pluridisciplinaire HAL, est destinée au dépôt et à la diffusion de documents scientifiques de niveau recherche, publiés ou non, émanant des établissements d'enseignement et de recherche français ou étrangers, des laboratoires publics ou privés. 


\title{
LES ETUDIANTS ET LE CONTRAT PREMIERE EMBAUCHE
}

\author{
Sébastien Michon, associé au Groupe de sociologie politique européenne (PRISME-GSPE, CNRS \\ UMR 7012).
}

\begin{abstract}
Résumé : Une enquête par questionnaire réalisée à Strasbourg auprès d'étudiants permet de rendre compte des liens entre attitudes à l'égard du Contrat première embauche (CPE) et caractéristiques sociopolitiques. Les réponses des étudiants témoignent de l'importance de dispositions à opiner politiquement et à se mobiliser (ou pas), développées au cours des socialisations primaires. Les analyses invitent également à ne pas négliger le contexte d'étude comme matrice socialisatrice à la mobilisation.
\end{abstract}

Cet article souhaite apporter des éléments de connaissance et d'explication sur la distribution des attitudes des étudiants à l'égard du Contrat première embauche (CPE), ainsi que sur le passage à la mobilisation contre ce projet début $2006^{1}$. Quels étaient les étudiants favorables, défavorables, mobilisés, non mobilisés ? Comment l'expliquer ? En tenant compte de ceux qui n'étaient pas mobilisés et en incluant un ensemble de variables politiques, sociales et scolaires, cette contribution à l'analyse des mouvements sociaux étudiants est complémentaire d'autres recherches en cours ${ }^{2}$. On souhaite ici adopter une approche en termes de dispositions et, partant, donner des éléments d'explication des pratiques et des prises de position des étudiants sur le CPE par leurs trajectoires sociales. Ceux-ci ayant incorporé, au cours de leurs socialisations primaires au sein de leur famille, non seulement des croyances et des schèmes de compréhension du monde social, mais aussi des manières de faire («se mobiliser ») en fonction desquels ils agissent ensuite ${ }^{3}$. Cependant, il s'agit aussi de prendre en compte la pluralité de modes de socialisation ${ }^{4}$, en précisant les mécanismes de l'activation des dispositions par le contexte d'étude.

Début décembre 2006 - quelques mois donc après le mouvement -, 519 étudiants à Strasbourg ont complété un questionnaire construit à partir de 78 variables ${ }^{5}$. Diffusé avec l'aide d'élèves de deuxième année de la faculté de sociologie dans le cadre d'un enseignement sur les méthodes d'enquête, il aborde un ensemble d'indicateurs des attitudes à l'égard du $\mathrm{CPE}$, du rapport à la politique des étudiants et de leurs parents, ainsi que de leurs caractéristiques scolaires et sociodémographiques. Afin de prendre quelque peu en compte la variété du contexte étudiant, l'échantillon est composé de quatre filières de l'enseignement supérieur avec des profils différents ${ }^{6}$ : droit, sciences (qui regroupent ici mathématiques, physique, chimie et sciences de la vie), sociologie et Institut d'Études Politiques (IEP) ; l'IEP accueille des étudiants beaucoup plus favorisés socialement et scolairement que la sociologie, les sciences sont plus masculinisées que le droit ou la sociologie. Précisons que le but n'est pas ici d'effectuer un sondage sur le taux de participation des étudiants aux manifestations contre le CPE à partir d'un échantillon représentatif d'un ou plusieurs critères (sexe, âge, origine sociale...), mais de mettre en évidence des écarts suivant différentes variables au moyen de techniques statistiques appropriées (tris croisés, analyses factorielles, classifications hiérarchiques, régressions logistiques). L'interrogation s'est faite directement sur les lieux d'étude, soit au début ou à la fin d'enseignements avec l'introduction d'un enseignant

\footnotetext{
${ }^{1}$ Pour un compte-rendu de la mobilisation, cf. Collectif 4 bis, 2007.

${ }^{2}$ Geay, Bodin, Camus, 2007 ; Nicourd, 2007.

${ }^{3}$ Bourdieu, 1980.

${ }^{4}$ Passeron, de Singly, 1984 ; Lahire, 1998.

5 À Strasbourg, le mouvement anti-CPE, comme dans les autres villes universitaires, s'est prolongé quelques semaines. L’Université des sciences humaines a été occupée. Les assemblées générales ont réuni plusieurs centaines d'étudiants, les manifestations plusieurs milliers.

${ }^{6}$ Grignon, 2000.
} 
(comme à l'IEP) ${ }^{7}$, soit devant les salles de classe avant ou après les cours (pour les trois autres filières). Si en raison de cette différence dans le mode d'interrogation, les étudiants n'avaient pas exactement les mêmes probabilités de répondre, il convient de souligner les très faibles taux de refus quelle que soit la filière. Au final, l'échantillon rassemble : 149 étudiants de l'IEP, 137 de sociologie, 110 de droit, 123 de sciences.

Les étudiants interrogés sont favorables au CPE à 27\% (64\% défavorables, 9\% sans opinion), c'est-à-dire dans des proportions supérieures à l'ensemble des 18-24 ans, à 16\% opposés à son retrait ${ }^{8}$. Les résultats présentés doivent évidemment être appréhendés au prisme de la composition de l'échantillon. Pour ce qui est de la mobilisation, un peu plus d'un étudiant sur deux n'a ici participé à aucune manifestation, 13\% à une, 24\% de deux à quatre, $12 \%$ à cinq et plus. Une ventilation proche pour les Assemblées générales (AG) : 52\% n'ont été à aucune, $19 \%$ à une ou deux, $17 \%$ de trois à neuf, $12 \%$ à dix et plus. Tous les étudiants défavorables n'ont donc pas été actifs dans le mouvement. Enfin, 23\% étaient pour le blocage des cours et $13 \%$ ont participé à des «actions ${ }^{9}$ », pour reprendre le terme employé par les étudiants mobilisés. Une distribution fortement clivée par leurs caractéristiques sociopolitiques et scolaires.

\section{L'ESPACE DES ATTITUDES A L'EGARD DU CPE}

Une analyse des correspondances multiples (ACM) effectuée à partir des principales questions et des 519 répondants, permet de mener une première exploration de la répartition des réponses. L'un des apports de ce type d'analyse est de proposer une représentation graphique qui associe les réponses suivant leur proximité (figure 1). Les étudiants se différencient dans leurs réponses sur le CPE selon leurs caractéristiques politiques, scolaires et sociales.

\footnotetext{
Une analyse des correspondances multiples

Le principe de cette technique statistique exploratoire est de mettre en correspondance un ensemble de variables. En étudiant les proximités et les distinctions entre les variables et les groupes, elle permet de décrire les principes de structuration des réponses relatives au CPE par rapport aux caractéristiques politiques, scolaires et sociales des étudiants, en distribuant les principales liaisons sur des axes. De manière habituelle, on détaillera ici les deux axes les plus significatifs qui, pour reprendre le vocabulaire technique, rendent compte de $6,30 \%$ et $4,55 \%$ de l'inertie totale; ce qui est loin d'être négligeable au regard du nombre de variables (16) et de modalités (88) insérées dans le modèle. Le premier facteur explicatif (axe 1) distribue les individus en fonction des positionnements sur le CPE et l'axe gauche/droite des étudiants et de leurs parents, ainsi que de l'origine sociale. Pour le résumer très brièvement, s'opposent ici les étudiants favorables au CPE, de droite, plus souvent à l'IEP ou en droit, d'origine sociale plutôt élevée notamment d'un point de vue économique, à ceux qui y sont défavorables, de gauche, plutôt scolarisés en sociologie et moins favorisés socialement. L'axe 2 fait état d'une opposition entre modalités extrêmes (les favorables et les défavorables au CPE, à droite ou à gauche, d'une origine sociale élevée ou non) et modalités médiantes (les sans opinions, ceux qui se placent au centre de l'axe gauche/droite, certaines catégories intermédiaires (artisans-commerçants ou techniciens)) - qui est aussi une opposition d'intérêt politique. On a là un effet Guttman dans le plan formé des axes 1 et 2 : les indicateurs les plus pertinents se répartissent se placent sur une parabole, ceux qui se situent à proximité de celle-ci étant mieux expliqués que ceux qui s'en éloignent.
}

\footnotetext{
${ }^{7}$ Merci à Frédéric Chateigner, Michel Daccache, Karim Fertikh et Audrey Freyermuth.

${ }^{8}$ Sondage CSA/Le Parisien Aujourd'hui en France/I-Télé réalisé le 21-22 mars 2006.

${ }^{9}$ À Strasbourg, entre autres : occupations diurnes et nocturnes de locaux (Université des sciences humaines pendant plusieurs semaines, l'École nationale d'administration pendant une heure), blocage de trains, de tramways ou du pont de l'Europe entre Strasbourg et Kehl, subtilisation d'urnes lors des élections du CROUS.
} 
Cette ACM donne également la possibilité d'effectuer une classification hiérarchique ascendante, autre technique statistique qui repose sur la construction de catégories. En réalisant une partition des individus de sorte à ce que chaque catégorie soit non seulement la plus différente possible des autres, mais aussi la plus homogène en fonction de ses caractéristiques, la classification réalisée regroupe les étudiants interrogés en quatre classes quatre idéaux-types - fonction de leurs attitudes sur le CPE et leurs propriétés politiques, scolaires et sociales.

Premier groupe: les favorables au CPE. Politisés et de droite, à l'instar de leurs parents, ils sont fréquemment originaires des catégories supérieures de l'espace social, principalement du pôle économique ${ }^{10}$. Ces étudiants, optimistes quant à leur avenir, sont scolarisés dans les filières les plus prestigieuses (IEP, droit). Deuxième groupe : les sans opinions sur le CPE et leur orientation politique. Principalement étudiants en sciences, ils s'intéressent peu aux «choses » politiques - comme leurs parents d'ailleurs. Troisième groupe : les opposants au CPE non mobilisés. De gauche, plutôt en droit, ils se déclarent proches du PS et envisagent de voter pour S. Royal, ils ont été au plus à deux manifestations et AG. Pour partie boursiers, leurs parents sont employés, artisans-commerçants, cadres intermédiaires. Quatrième groupe: les mobilisés. Ils ont participé au mouvement (manifestations, AG) et forment trois sous-groupes.

Le premier rassemble les étudiants qui n'étaient pas les plus actifs. Ils ont certes été à plusieurs manifestations (trois à quatre) et AG (trois à neuf), sans néanmoins prendre part aux actions. Aux deux tiers étudiants en sociologie, se situant à la gauche du PS, avec des parents assez souvent cadres intermédiaires (enseignants notamment), ils se caractérisent par une forme de bonne volonté de gauche. Par rapport aux deux sous-groupes suivants, les filles y sont surreprésentées. Signe de la division sexuelle du travail militant ${ }^{11}$, la variable sexuée est particulièrement clivante pour ce qui est des pratiques militantes les plus actives : alors que les filles forment $60 \%$ de l'échantillon, elles ne représentent que $41 \%$ de ceux qui ont participé à cinq manifestations et plus, $38 \%$ à dix $\mathrm{AG}$ et plus, et $44 \%$ aux « actions ».

Les plus mobilisés, favorables au blocage des cours, se retrouvent dans les deux derniers sous-groupes. L'un est celui des concernés. Ce sont les étudiants parmi les plus modestes de l'échantillon (mère employée, père ouvrier, peu diplômés). Ces étudiants sont caractéristiques des générations « $80 \%$ au bac ${ }^{12} »$ : en sociologie (aux trois-quarts), boursiers (la moitié), salariés (un tiers), au capital scolaire peu élevé (un quart avec un bac technique ou professionnel). Faisant majoritairement état d'une socialisation familiale à gauche, voire même très à gauche, certains sont proches de la mouvance libertaire et révolutionnaire (CNT notamment).

L'autre est celui des héritiers de gauche, dans l'échantillon numériquement moins nombreux que ceux du précédent sous-groupe. Davantage enclins à adhérer à une définition légitime de la politique, ils sont moins à l'extrême gauche que les précédents : deux tiers se déclarent proches du PS, plus précisément «la gauche du PS » comme quelques uns l'ont signalé sur le questionnaire, ou comme l'indique leur positionnement en 2 sur l'axe gauche/droite $^{13}$. Très politisés, à l'instar de leurs parents, une partie adhère à un parti (Mouvement des jeunes socialistes (MJS)) et/ou à l'UNEF. Outre leur positionnement

\footnotetext{
${ }^{10}$ On fait ici référence à l'espace social construit par Bourdieu, 1979.

${ }^{11}$ Bargel, 2005.

12 Beaud, 2002.

${ }^{13}$ L'indicateur ici utilisé est la question habituelle des enquêtes sur les comportements politiques (Michelat, Simon, 1977) qui demande aux étudiants de se positionner sur un axe gauche/droite comprenant sept cases allant de l'extrême gauche à l'extrême droite : trois à gauche, trois à droite, une au centre - c'est-à-dire la quatrième. Deux autres possibilités sont offertes aux répondants : ne pas répondre ou cocher la case « ne sais pas ».
} 
politique, ces étudiants se distinguent par leur capital culturel élevé, que ce soit dans sa dimension héritée (leurs parents sont fréquemment professeurs du secondaire voire du supérieur) ou acquise (mention au bac, majoritairement scolarisés à l'IEP). Illustratif d'une adhésion au jeu scolaire et d'une sensibilisation précoce à certaines formes de participation et d'engagement, deux tiers ont été délégués de classe dans le secondaire. À noter les différences d'attitudes observées lors de la mobilisation entre les deux sous-groupes des étudiants mobilisés: les concernés prônant toujours plus «de radicalité » que les héritiers, pour reprendre leurs termes.

En conséquence, les techniques statistiques employées rendent compte de l'espace des attitudes à l'égard du CPE. Un espace clivé par les variables sociopolitiques. Des liaisons qu'il s'agit de détailler plus précisément.

\section{DES SOCIALISATIONS PRIMAIRES CLIVANTES}

\section{Le rapport au CPE politiquement situé}

D'un côté, se trouvent donc les étudiants favorables au CPE qui n'ont pas participé aux manifestations et aux actions contre le CPE, ni aux AG. Ils s'orientent à droite et envisagent de voter pour N. Sarkozy, F. Bayrou ou D. de Villepin ${ }^{14}$ au premier tour de l'élection présidentielle de 2007. De l'autre côté, les étudiants défavorables au CPE, qui ont massivement participé au mouvement. En dehors de quelques uns qui refusent de se situer sur l'axe gauche/droite proposé, ils choisissent surtout les positions les plus à gauche. Une liaison particulièrement forte entre les variables est effectivement celle entre opinions sur le CPE et opinions politiques : $25 \%$ des étudiants qui se situent à la droite de l'axe gauche/droite se déclarent opposés au $\mathrm{CPE}$, contre $86 \%$ pour ceux situés à gauche, $55 \%$ au centre et $64 \%$ de ceux qui choisissent la réponse «ne sais pas » (tableau 1).

Ceci dit, si pratiquement tous ceux qui se positionnent à gauche souhaitent le retrait $\mathrm{du}$ CPE (86\%), tous n'ont pas participé aux mobilisations et autres actions. Les manifestations et les AG ont plus fédéré que les blocages : 70\% des étudiants de gauche ont participé à au moins une manifestation (18\% des étudiants de droite, $37 \%$ du centre, $41 \%$ des «ne sais pas »), $62 \%$ à au moins une AG (30\% environ des étudiants de droite et du centre), seulement $20 \%$ à des actions (quasiment aucun au centre et à droite). Le blocage des cours ne faisait d'ailleurs pas l'unanimité parmi les opposants au projet puisqu'à gauche, seuls $38 \%$ l'ont approuvé. Des attitudes pendant le mouvement de fait non homogènes. Les plus actifs, qui souscrivaient le plus au blocage des cours sont les étudiants les plus à gauche, c'est-à-dire ceux en 1 ou 2 sur l'axe, soutiens des candidatures de O. Besancenot, A. Laguiller, M.-G. Buffet ou J. Bové à la présidentielle de 2007, qui ont voté non au référendum sur le Traité constitutionnel européen de 2005 (tableau 2).

L'orientation politique est clivante, tout comme l'intérêt politique. En ce sens, le deuxième axe de l'ACM particularise les sans opinions sur le CPE, qui sont également ceux sans positionnement politique et sans intérêt politique. En fait, les «pour » et les «contre » le CPE se différencient peu par leur niveau d'intérêt politique, ceux qui étaient favorables au CPE étant tout de même un peu plus politisés (deux tiers d'entre eux se déclarent intéressés contre la moitié des défavorables), les sans opinions sur le CPE beaucoup moins (un tiers).

Pour les étudiants, comme pour le reste de la population ${ }^{15}$, l'orientation politique et la politisation ne sont pas distribuées au hasard, mais variables suivant l'origine sociale et les

\footnotetext{
${ }^{14}$ Au moment de l'enquête, les candidats à la présidentielle n'étaient pas tous connus.

${ }^{15}$ Gaxie, 1978.
} 
caractéristiques politiques familiales, c'est-à-dire ici l'orientation politique des parents et leur familiarité avec la politique : $65 \%$ des étudiants de gauche ont un père à gauche ; $74 \%$ des étudiants intéressés par la politique ont un père intéressé par la politique ; 59\% des étudiants dont le père a des revenus mensuels inférieurs à 2250 euros se positionnent à la gauche de l'axe contre $12 \%$ avec des revenus supérieurs à 3000 euros; $65 \%$ des étudiants dont le père a un niveau de diplôme supérieur ou égal à bac+5 s'intéressent à la politique contre $48 \%$ avec un niveau inférieur au bac. Les fortes liaisons entre attitudes à l'égard du CPE et rapport à la politique sont en fait déterminées par les socialisations primaires.

\section{Effets d'héritage}

$\mathrm{Au}$ cours de leur enfance et leur adolescence, les étudiants ont développé des dispositions à produire telle ou telle opinion et à se mobiliser ou pas, des dispositions souvent mises en action lors du mouvement contre le CPE.

L'influence de la socialisation politique parentale est tout d'abord patente ; la famille constitue de facto un lieu «où se façonnent et se transmettent les valeurs et les modèles culturels entre les générations [...] à partir desquels tout citoyen va établir ses liens élémentaires au monde politique ${ }^{16} \gg$. En effet, les variations ne sont pas du tout négligeables : $54 \%$ des étudiants favorables au CPE déclarent avoir un père de droite, $19 \%$ seulement des défavorables; les parents de près de trois-quarts des étudiants qui ont manifesté cinq fois et plus, et deux tiers de ceux qui approuvaient le blocage se situent à gauche; enfin, les étudiants avec des positions affirmées sur le CPE (les pour et les contre) ont les parents les plus politisés, au contraire des sans opinions : 60\% environ des étudiants qui se déclarent pour ou contre le CPE considèrent que leur père s'intéresse à la politique, contre seulement $45 \%$ des étudiants sans opinions sur le CPE.

La classe sociale déterminant pour partie les formes de socialisation ${ }^{17}$, il n'est guère étonnant de constater une distribution différentielle des attitudes sur le CPE selon l'origine sociale : y être favorable étant surtout le fait des plus favorisés socialement. Les pères des étudiants pour le maintien du CPE sont davantage chefs d'entreprise ou cadres supérieurs du privé, avec des diplômes supérieurs ou égaux à bac +5 et des revenus mensuels supérieurs à 3000 ou 4500 euros. Ceux des opposants sont en revanche plus fréquemment d'origines modestes, peu ou pas diplômés, ouvriers, employés, aux salaires les moins conséquents (inférieurs à 1500 euros). À ces étudiants se joignent des enfants de cadres intermédiaires du public (enseignants ou éducateurs notamment). Plus précisément, $42 \%$ de ceux dont le père est chef d'entreprise, cadre supérieur dans le privé ou exerce une profession libérale, se prononcent en faveur du CPE, contre moins de $10 \%$ des fils d'ouvriers. Des écarts qui se retrouvent avec les positions sur le blocage des cours et la participation à des actions. Si $23 \%$ de l'échantillon approuvent le blocage des cours, tel est le cas de : $48 \%$ des fils d'ouvriers (seulement $12 \%$ de cadres supérieurs du privé), $42 \%$ de ceux dont les revenus du père sont inférieurs à 1500 euros (25\% entre 2250 et 3000 euros, environ $10 \%$ au-delà). La distribution ne suit cependant pas toujours une opposition en termes de stratification sociale : ceux avec les pères les plus diplômés (39\% pour ceux dont les parents ont un niveau supérieur ou égal à bac+5) sont certes les plus favorables au CPE, mais les enfants de bac+3/4 le sont cependant moins (27\%) que les étudiants dont le père est doté d'un CAP/BEP (31\%). On doit ici évoquer, non seulement des variations suivant la position sociale héritée (entre favorisés et moins favorisés), mais aussi une distinction entre public et privé : $36 \%$ des enfants de cadres

\footnotetext{
${ }^{16}$ Muxel, 2001, p. 52.

${ }^{17}$ Bernstein, 1975.
} 
intermédiaires du privé sont pour, seulement $11 \%$ pour ceux du public. C'est là une différence de structure des propriétés sociales ${ }^{18}$ : les plus dotés en capital économique - plutôt de droite - sont plus favorables que ceux avec des ressources sociales héritées davantage culturelles (enfants d'enseignants, d'éducateurs) - plutôt de gauche.

Ainsi, l'enquête met en exergue les effets des socialisations primaires sur les attitudes à l'égard du CPE. Néanmoins, les étudiants ont été confrontés à d'autres modes de socialisations, scolaires notamment.

\section{LE CONTEXTE D'ETUDE A NE PAS NEGLIGER}

De prime abord, si le contexte d'étude en tant que cadre de socialisation ne doit pas être minimisé ${ }^{19}$, au regard des fortes dépendances entre comportements politiques et socialisations primaires, son effet sur le rapport au CPE ne va pas de soi.

Les réponses des étudiants sur le CPE sont tout d'abord particulièrement différenciées suivant les variables scolaires. Les opinions sur le CPE se répartissent inégalement suivant le capital scolaire : seuls $10 \%$ des étudiants dotés d'un bac technique ou professionnel - surtout scolarisés en sociologie - sont pour le maintien du CPE contre, on le rappelle, $27 \%$ de l'échantillon. Ceux qui ont le moins de chances objectives de réussite dans le supérieur ${ }^{20}$, qui étaient les plus susceptibles d'être confrontés au CPE, font effectivement partie des plus opposés et des plus actifs : $52 \%$ des bacs techniques et professionnels et $32 \%$ de ceux qui n'ont pas eu de mention au bac étaient pour le blocage des cours (contre 23\% sur l'ensemble). Mais, plus que le capital scolaire, la filière d'étude clive davantage encore les comportements. Les étudiants de l'IEP et de droit sont les plus positifs à l'égard du CPE, ceux de sociologie le moins, ceux en sciences les plus indifférents (la moitié des sans opinions se trouvent en sciences) (tableau 3). Et surtout, les apprentis sociologues ont été les plus actifs dans le mouvement : quatre cinquièmes ont participé à au moins un AG (contre au plus $40 \%$ dans les trois autres filières), deux tiers à au moins une manifestation (contre $41 \%$ à $44 \%$ dans les trois autres filières) ; $42 \%$ étaient pour le blocage des cours (contre 16\% à 17\%).

De fortes variations donc, que l'on se doit de tempérer au regard des dépendances entre les variables explicatives. Les étudiants de l'IEP étant les plus favorisés scolairement et socialement contrairement à ceux de sociologie (tableau 4), on peut légitimement se demander si ces résultats ne sont pas la seule conséquence des effets de la socialisation politique parentale et de l'origine sociale précédemment mis en évidence. Cependant, deux types d'opérations statistiques font tout de même état d'un effet des filières d'étude, notamment pour ce qui est des opinions sur le blocage des cours et de la participation aux manifestations.

Des régressions logistiques, d'une part. Elles permettent de mesurer, «toutes choses égales par ailleurs », l'effet propre de la filière. Les trois modèles (tableau 5), explicatifs des opinions sur le CPE et le blocage des cours ainsi que de la participation à une manifestation, reposent sur la filière d'étude, l'orientation politique du père et le niveau de diplôme de celui$\mathrm{ci}^{21}$. Chacun d'entre eux spécifie le positionnement politique du père comme la variable la plus explicative. Néanmoins, alors que pour chaque modèle, le niveau de diplôme du père n'est significatif que pour la participation à une manifestation, la filière d'étude l'est

\footnotetext{
${ }^{18}$ Bourdieu, 1979.

${ }^{19}$ Millet, 2003.

${ }^{20}$ Blöss, Erlich, 2000 ; Beaud, 2002.

${ }^{21}$ Le niveau de diplôme a été préféré aux revenus en raison de taux de non-réponses moins élevés.
} 
toujours : l'IEP et surtout la sociologie ${ }^{22}$, les étudiants de sociologie ont beaucoup plus de chances d'être contre le CPE et surtout favorables au blocage des cours, ainsi que d'avoir participé à une manifestation.

$\mathrm{Si}$, compte tenu des dépendances entre les variables explicatives insérées, on pourrait évoquer les limites des modèles de régression, une analyse multivariée à partir de croisements de variables sur des sous-populations conforte néanmoins les précédentes observations. Parmi les étudiants de sociologie dont le père est de gauche, $4 \%$ sont favorables au CPE en sociologie (contre $22 \%$ à l'IEP, $25 \%$ en droit, $13 \%$ en sciences), $58 \%$ approuvent le blocage des cours (contre 33\% à l'IEP, 35\% en droit, 29\% en sciences). De la sorte, les étudiants de sociologie présentent des comportements plus spécifiques. Leur positionnement dominé dans l'espace universitaire et leurs probabilités moins fortes d'accéder à des positions dominantes dans l'espace social sont certes à prendre en compte. En même temps, l'on ne peut pas rejeter une socialisation par le contexte d'études. La filière d'étude favorise l'activation et l'acquisition de dispositions non seulement à la politisation ${ }^{23}$, mais aussi à la mobilisation. Elle forme un agent possible de socialisation au mouvement susceptible de favoriser une participation active. En ce sens, les observations menées laissent envisager, à Strasbourg, des étudiants de sociologie davantage au contact du mouvement, par l'intermédiaire de leurs camarades de cours et de la proximité spatiale avec les lieux de réunion des étudiants: plusieurs leaders du mouvement étudiaient en sociologie et la plupart des AG se déroulaient au sein même de leur université. Le rôle des enseignants, plus mobilisés que dans les autres filières, n'est pas non plus à négliger. Autant de vecteurs susceptibles d'encourager la mobilisation, et par là l'accès à l'arène socialisatrice que représente le mouvement lui-même.

L'objectivation statistique permet ainsi de conforter ou de corriger des intuitions, des impressions, que d'aucuns ont pu formuler, mobilisé ou non, observateur proche ou lointain. Plusieurs techniques statistiques mettent en exergue les liens entre les attitudes par rapport au CPE et les variables politiques, sociales et scolaires. Les opinions et les pratiques des étudiants interrogés sont liées aux dispositions qu'ils ont développées dès leur enfance, des dispositions activées lors du mouvement. L'enquête renseigne également sur les effets du contexte d'étude. Dans le cadre de l'enquête, la sociologie se présente comme un contexte plus favorable à la mobilisation : les étudiants y ont été plus prompts à se prononcer pour le blocage des cours et à manifester. Le rôle du contexte d'action ne doit pas être minimisé dans l'analyse des mouvements étudiants. Bien évidemment, d'autres enquêtes plus qualitatives restent à mener afin de préciser celui-ci.

\section{Bibliographie}

BARGEL L., «La socialisation politique sexuée : apprentissage des pratiques politiques et normes de genre chez les jeunes militant-e-s », Nouvelles Questions Féministes, vol. 24, n 3, 2005, pp. 36-49.

BEAUD S., 80\% au bac... et après ? Les enfants de la démocratisation scolaire, La découverte, Paris, 2002.

BERNSTEIN B., Langage et classes sociales, Minuit, Paris, 1975.

BLOSS T., ERLICH V., «Les nouveaux «acteurs » de la sélection universitaire : les bacheliers technologiques en question », Revue française de sociologie, vol. 41, n 4, 2000, pp. 747-775.

BOURDIEU P., La distinction, critique sociale du jugement, Minuit, Paris, 1979.

\footnotetext{
${ }^{22}$ Les odds-ratio, les « rapports de chances », s'élèvent, pour la sociologie, à 1,330 pour l'opinion sur le CPE, 3,712 concernant le blocage des cours, et 3,861 pour la participation à une manifestation (tableau 5).

${ }^{23}$ Michon, 2006.
} 
BOURDIEU P., Le sens pratique, Minuit, Paris, 1980.

COLLECTIF 4 bis, Le CPE est mort... pas la précarité! Retour sur le printemps étudiant 2006, Éd. Syllepse, Paris, 2007.

GAXIE D., Le cens caché, Éd du Seuil, Paris, 1978.

GEAY B., BODIN R., CAMUS J., « Jeunesse, travail et action collective : le cas des étudiants français mobilisés contre le Contrat Première Embauche », communication aux «Rencontres Jeunes et Sociétés en Europe et autour de la Méditerranée », Marseille, octobre 2007.

GRIGNON C. (dir.), Les conditions de vie des étudiants, enquête OVE, Presses Universitaires de France, Paris, 2000 .

LAHIRE B., L'homme pluriel. Les ressorts de l'action, Nathan, Paris, 1998.

MICHELAT G., SIMON M., Classe, religion, et comportement politique, Presses de Sciences Po, Paris, 1977.

MICHON S., Études et politique : les effets de la carrière étudiante sur la socialisation politique, Thèse de doctorat en sociologie, Université Strasbourg 2, 2006.

MILLET M., Les étudiants et le travail universitaire, Presses Universitaires de Lyon, Lyon, 2003.

MUXEL A., L'expérience politique des jeunes, Presses de Sciences Po, Paris, 2001.

NICOURD S., "S'engager dans un mouvement étudiant: les conditions d'une socialisation politique », communication aux « Rencontres Jeunes et Sociétés en Europe et autour de la Méditerranée », Marseille, octobre 2007.

PASSERON J.-C., SINGLY F. de, «Différences dans la différence : socialisation de classe et socialisation sexuelle », Revue française de science politique, vol. 34, n 1, 1984, pp. 48-73.

\section{Tableaux et figures}

Figure 1 : Analyse des correspondances multiples sur les attitudes des étudiants à l'égard du CPE et leurs propriétés sociopolitiques (la parabole ajoutée sur le dessin vise à rendre compte de l'effet Guttman).

Champ : les étudiants interrogés au cours de l'enquête.

Source : enquête Strasbourg décembre 2006.

Légende: Filière d'étude; Attitudes par rapport au CPE; autres variables sociopolitiques; orientation politique. («int pol »: lire « intérêt politique »; «p » : lire «père »; «NSP » : lire « ne sais pas »). 


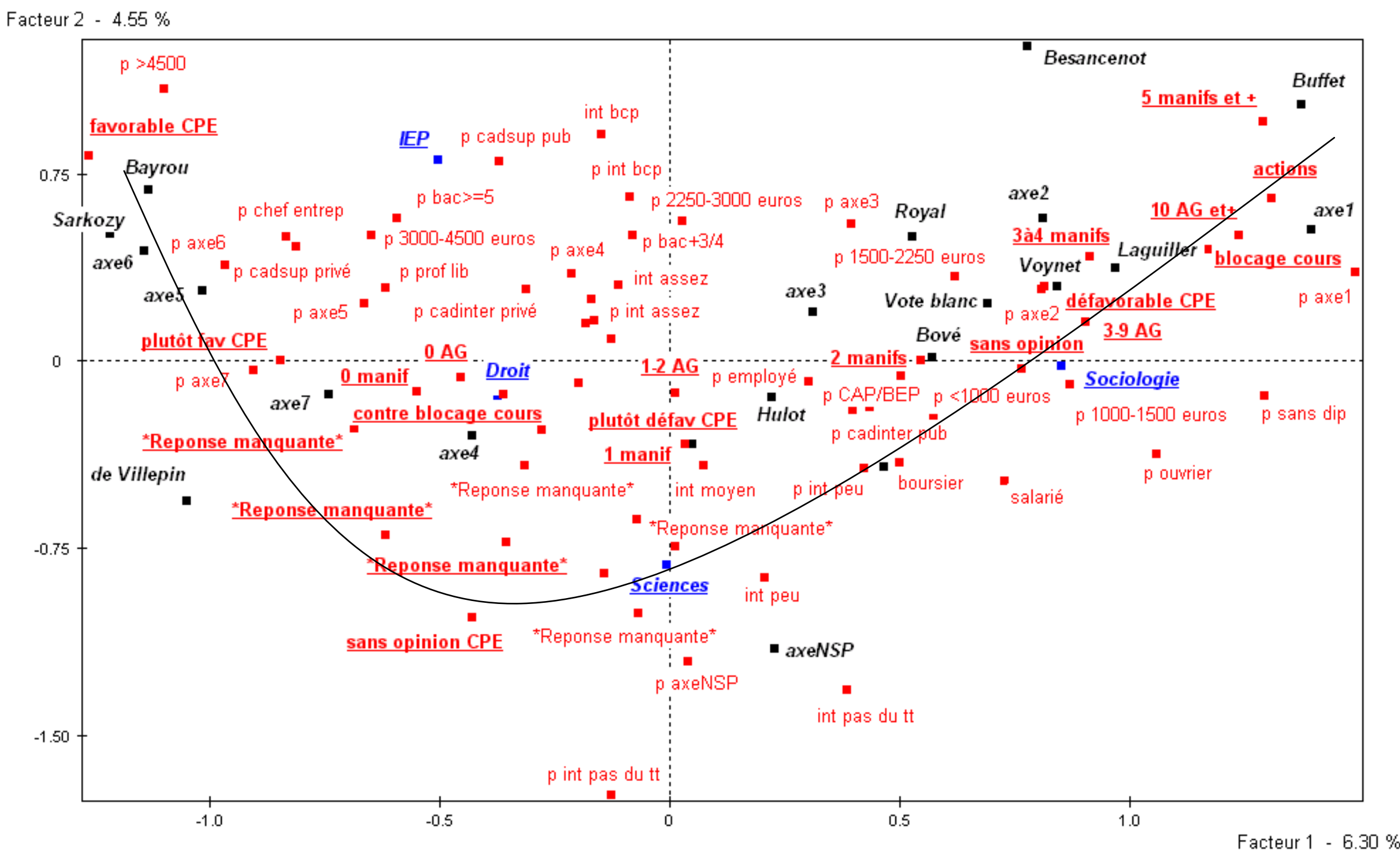


Tableau 1 : Opinions par rapport au CPE selon le positionnement sur l'axe gauche/droite ${ }^{24}(\mathbf{p}<0,001)$.

\begin{tabular}{|l|c|c|}
\hline & $\%$ (effectifs) & $\%$ d'étudiants défavorables au CPE \\
\hline Extrême gauche (case 1) & $4 \%(23)$ & $96 \%$ \\
\hline Gauche (case 2) & $15 \%(80)$ & $95 \%$ \\
\hline Centre gauche (case 3) & $25 \%(127)$ & $79 \%$ \\
\hline Centre (case 4) & $16 \%(84)$ & $55 \%$ \\
\hline Centre droit (case 5) & $15 \%(77)$ & $30 \%$ \\
\hline Droite (case 6) & $7 \%(35)$ & $17 \%$ \\
\hline Extrême droite (case 7) & $1 \%(6)$ & $0 \%$ \\
\hline Case « Ne sais pas » & $15 \%(78)$ & $64 \%$ \\
\hline Non réponse & $2 \%(9)$ & $56 \%$ \\
\hline Total & $100 \%(519)$ & $73 \%$ \\
\hline
\end{tabular}

Champ : les étudiants interrogés au cours de l'enquête.

Lecture : sur les 23 étudiants interrogés (soit 4\%) qui se positionnent à l'extrême gauche (la case la plus à gauche sur l'axe proposé), $96 \%$ se déclarent défavorables au CPE.

Source : enquête Strasbourg décembre 2006.

Tableau 2 : Attitudes des étudiants de gauche à diverses actions selon leur positionnement sur l'axe.

\begin{tabular}{|c|c|c|c|c|}
\hline & $\begin{array}{l}\text { Etre favorable au } \\
\text { blocage des cours }\end{array}$ & $\begin{array}{l}\text { Avoir participé à } 5 \\
\text { manifestations et plus }\end{array}$ & $\begin{array}{l}\text { Avoir participé à } \\
10 \text { AG et plus }\end{array}$ & $\begin{array}{l}\text { Avoir participé à des } \\
\text { actions } \\
\text { (blocage d'Université, de } \\
\text { tram, occupation de locaux) }\end{array}$ \\
\hline Valeur de p & 0,02 & $\mathrm{NS}^{25}$ & $<0,001$ & NS \\
\hline $\begin{array}{c}\text { Extrême } \\
\text { gauche (case } \\
1) \\
(23)\end{array}$ & $70 \%$ (16) & $41 \%(9)$ & $32 \%(7)$ & $39 \%(9)$ \\
\hline $\begin{array}{c}\text { Gauche (case } \\
\text { 2) } \\
(80)\end{array}$ & $48 \%(38)$ & $23 \%(18)$ & $22 \%$ & $24 \%$ (19) \\
\hline $\begin{array}{c}\text { Centre- } \\
\text { gauche (case } \\
\text { 3) } \\
(127)\end{array}$ & $25 \%$ & $15 \%$ (19) & $14 \%$ (18) & $15 \%$ (19) \\
\hline
\end{tabular}

Champ : les étudiants qui se sont positionnés sur la gauche de l'axe.

Lecture : $70 \%$ des étudiants qui se positionnent sur la première case de l'axe étaient favorables au blocage des cours, contre $48 \%$ de ceux sur la case $2,25 \%$ sur la case 3 .

Source : enquête Strasbourg décembre 2006.

\footnotetext{
${ }^{24}$ La question sur l'axe gauche/droite demande aux enquêtés de se positionner sur une échelle de sept cases allant de l'extrême gauche à l'extrême droite (trois cases à gauche, trois à droite, une au centre). Afin de limiter quelque peu l'imposition de problématique de cet axe, il est également possible de cocher une case «ne sais pas ».

${ }^{25}$ Non significatif
} 
Tableau 3 : Opinions sur le CPE selon la filière d'étude $(p<0,001)$.

\begin{tabular}{|l|c|c|c|c|}
\hline & Favorables & Défavorables & Sans opinion & Total \\
\hline IEP & $44 \%(63)$ & $53 \%(78)$ & $3 \%(5)$ & $100 \%(146)$ \\
\hline Sociologie & $13 \%(17)$ & $83 \%(114)$ & $4 \%(6)$ & $100 \%(137)$ \\
\hline Droit & $30 \%(32)$ & $59 \%(64)$ & $11 \%(12)$ & $100 \%(108)$ \\
\hline Sciences & $23 \%(28)$ & $60 \%(73)$ & $17 \%(21)$ & $100 \%(122)$ \\
\hline
\end{tabular}

Champ : les étudiants interrogés au cours de l'enquête.

Lecture : $44 \%$ des étudiants interrogés à l'IEP sont favorables au CPE.

Source : enquête Strasbourg décembre 2006.

Tableau 4 : Caractéristiques politiques et sociales selon la filière d'étude $(\mathbf{p}<0,001)$.

\begin{tabular}{|c|c|c|c|c|c|}
\hline & Boursiers & Salariés & $\begin{array}{c}\text { Père avec un } \\
\text { diplôme du supérieur }\end{array}$ & $\begin{array}{c}\text { Père avec des revenus } \\
\text { mensuels supérieurs à } \\
3000 \text { euros }\end{array}$ & $\begin{array}{c}\text { Père avec une } \\
\text { profession supérieure } \\
\text { (chef d'entreprise, } \\
\text { cadre supérieur ou } \\
\text { profession libérale) }\end{array}$ \\
\hline IEP & $15 \%(22)$ & $2 \%(4)$ & $77 \%(96)$ & $48 \%(46)$ & $50 \%(70)$ \\
\hline Sociologie & $35 \%(48)$ & $29 \%(40)$ & $32 \%(35)$ & $10 \%(9)$ & $17 \%(22)$ \\
\hline Droit & $26 \%(29)$ & $13 \%(14)$ & $58 \%(43)$ & $25 \%(12)$ & $36 \%(35)$ \\
\hline Sciences & $32 \%(39)$ & $15 \%(19)$ & $47 \%(40)$ & $25 \%(14)$ & $25 \%(28)$ \\
\hline
\end{tabular}

Champ : les étudiants interrogés au cours de l'enquête.

Lecture : $15 \%$ des étudiants interrogés à l'IEP sont boursiers.

Source : enquête Strasbourg décembre 2006. 
Tableau 5 : Modèles de régression logistique sur le fait d'être défavorable au CPE (Modèle 1), favorable au blocage des cours (Modèle 2), et d'avoir manifesté contre le CPE (Modèle 3).

\begin{tabular}{|c|c|c|c|c|c|c|c|c|c|}
\hline & \multicolumn{3}{|c|}{ Modèle 1} & \multicolumn{3}{|c|}{ Modèle 2} & \multicolumn{3}{|c|}{ Modèle 3} \\
\hline & $\begin{array}{l}\text { Odds- } \\
\text { ratio }\end{array}$ & $\begin{array}{c}\text { Etre } \\
\text { défavorable au } \\
\text { CPE }\end{array}$ & Significativité & $\begin{array}{l}\text { Odds- } \\
\text { ratio }\end{array}$ & $\begin{array}{c}\text { Etre } \\
\text { favorable } \\
\text { au } \\
\text { blocage }\end{array}$ & Significativité & $\begin{array}{c}\text { Odds- } \\
\text { ratio }\end{array}$ & $\begin{array}{c}\text { Avoir } \\
\text { manifesté }\end{array}$ & Significativité \\
\hline Valeur de c & & 0,704 & & & 0,787 & & & 0,737 & \\
\hline \multicolumn{10}{|l|}{ Filière } \\
\hline IEP & 0,512 & $-0,5387$ & 0,003 & 1,021 & $-0,4411$ & 0,06 & 1,854 & $-0,4411$ & NS \\
\hline Sociologie & 1,330 & 0,4168 & 0,01 & 3,712 & 0,8492 & $<0,0001$ & 3,861 & 0,8492 & $<0,0001$ \\
\hline Droit & 0,868 & $-0,010$ & NS & 1,676 & 0,0543 & NS & 1,372 & 0,0543 & NS \\
\hline Sciences & Réf. & Réf. & Réf. & Réf. & Réf. & Réf. & Réf. & Réf. & Réf. \\
\hline \multicolumn{10}{|l|}{$\begin{array}{l}\text { Orientation } \\
\text { politique } \\
\text { du père }\end{array}$} \\
\hline \multirow{3}{*}{$\begin{array}{r}\text { Gauche } \\
\text { Centre } \\
\text { Droite }\end{array}$} & 3,165 & 0,8297 & $<0,0001$ & 3,345 & 1,2151 & $<0,0001$ & 1,892 & 1,2151 & $<0,0001$ \\
\hline & Réf. & Réf. & Réf. & Réf. & Réf. & Réf. & Réf. & Réf. & Réf. \\
\hline & 0,756 & $-0,6017$ & 0,0007 & 0,318 & $-1,1392$ & 0,0002 & 0,421 & $-1,1392$ & $<0,0001$ \\
\hline $\mathrm{Ne}$ sait pas & 1,517 & 0,0944 & NS & 0,913 & $-0,0836$ & NS & 0,848 & $-0,0836$ & NS \\
\hline \multicolumn{10}{|l|}{$\begin{array}{l}\text { Niveau de } \\
\text { diplôme du } \\
\text { père }\end{array}$} \\
\hline$<$ bac & 0,824 & 0,0116 & NS & 0,745 & $-0,1228$ & NS & 0,521 & $-0,1228$ & NS \\
\hline$>$ bact2 & Réf. & Réf. & Réf. & Réf. & Réf. & Réf. & Réf. & Réf. & Réf. \\
\hline & 0,655 & $-0,2174$ & NS & 0,802 & $-0,0491$ & NS & 0,290 & $-0,0491$ & 0,0005 \\
\hline
\end{tabular}

Champ : 477 étudiants interrogés au cours de l'enquête qui ont répondu aux questions retenues.

Lecture : La modalité que l'on cherche à expliquer sur le premier modèle est « être défavorable au CPE ». Le modèle utilisé ici est de type logit. Une valeur statistiquement significative et positive indique que l'on est en présence d'un facteur qui accroît les chances pour un étudiant d'être défavorable au CPE, toutes autres choses égales par ailleurs. Une valeur statistiquement significative et négative indique que l'on est en présence d'un facteur qui diminue les chances pour un étudiant d'être défavorable au CPE. La valeur de c est un indicateur d'évaluation de la qualité du modèle : plus il est élevé, meilleur est le modèle (c dépend du pourcentage des pairs concordantes). L'odds-ratio est l'exponentielle du coefficient $\beta$ d'ajustement logistique, c'est-à-dire le coefficient multiplicateur associé à une modalité par rapport à la modalité de référence. Les odds-ratio, les « rapports de chances », sont calculés par rapport à la modalité de référence. La situation de référence est un étudiant en sciences, dont le père se positionne politiquement au centre et dont le diplôme le plus élevé est d'un niveau bac/bac+2. Avoir un père de gauche et être scolarisé en sociologie est favorable à l'opposition au CPE, au contraire du fait d'avoir un père de droite avec un niveau de diplôme supérieur à bac+2.

Source : enquête Strasbourg décembre 2006. 